Reproducing Race 
This page intentionally left blank 


\section{Reproducing Race}

AN ETHNOGRAPHY OF PREGNANCY

AS A SITE OF RACIALIZATION

Khiara M. Bridges

甲

UNIVERSITY OF CALIFORNIA PRESS

BERKELEY LOS ANGELES LONDON 
University of California Press, one of the most distinguished university presses in the United States, enriches lives around the world by advancing scholarship in the humanities, social sciences, and natural sciences. Its activities are supported by the UC Press Foundation and by philanthropic contributions from individuals and institutions. For more information, visit www.ucpress.edu.

University of California Press

Berkeley and Los Angeles, California

University of California Press, Ltd.

London, England

(C) 20 Ir by The Regents of the University of California

Library of Congress Cataloging-in-Publication Data

Bridges, Khiara M.

Reproducing race : an ethnography of pregnancy as a site of racialization / Khiara M. Bridges. p. cm.

Includes bibliographical references and index.

ISBN 978-0-520-26894-4 (hardcover : alk. paper) — ISBN 978-0-520-26895-I (paperback : alk. paper) I. Alpha Hospital (New York, N.Y.) 2. Hospitals—Maternity services-New York (State)New York. 3. Discrimination in medical care-New York (State)-New York. 4. MinoritiesMedical care-New York (State)—New York. I. Title.

[DNLM: I. Alpha Hospital (New York, N.Y.)

2. Maternal Health Services—standards—New York City. 3. Maternal Health Servicesstandards-United States. 4. Anthropology, Cultural-New York City. 5. Anthropology, Cultural-United States. 6. Healthcare Disparities-New York City. 7. Healthcare

Disparities—United States. 8. Hospitals, Municipal—standards—New York City.

9. Hospitals, Municipal—standards—United States. Io. Prenatal Care-New York City.

II. Prenatal Care-United States. I2. Socioeconomic Factors-New York City.

I3. Socioeconomic Factors_-United States. WA 3io AN7]

RG50I.U6B75 2011

362.19'82009747-dc22

2010041690

Manufactured in the United States of America

$\begin{array}{lllllllllll}20 & \text { I9 } & \text { I8 } & \text { I7 } & \text { I6 } & \text { I5 } & \text { I4 } & \text { I3 } & \text { I2 } & \text { II } \\ \text { IO } & 9 & 8 & 7 & 6 & 5 & 4 & 3 & 2 & \text { I } & \end{array}$

This book is printed on Cascades Enviro Ioo, a Ioo\% post consumer waste, recycled, de-inked fiber. FSC recycled certified and processed chlorine free. It is acid free, Ecologo certified, and manufactured by BioGas energy. 
To Clive R. Bridges and Deborah A. Bridges, who have loved me flawlessly 
This page intentionally left blank 